\title{
ANCA-associated vasculitis: diagnosis and treatment in the elderly
}

\author{
Robin Parry, Simon Sherwin, Valerie Fletcher, Pippa Medcalf
}

\begin{abstract}
Summary
We report the successful treatment of three patients with ANCA-associated vasculitis aged 79,80 , and 80 years. We also review other published reports of treatment in the elderly and discuss complications of treatment with emphasis on elderly patients.
\end{abstract}

Keywords: ANCA, vasculitis, microscopic polyangiitis, Wegener's granulomatosis, elderly

The systemic vasculitides are rare disorders. Their classification is based on histopathology, size of vessel involved and clinical symptomatology. Many patients present with overlapping features of different vasculitides. A subgroup of vasculitides (Wegener's granulomatosis, microscopic polyarteritis, Churg Strauss syndrome) is strongly associated with circulating anti-neutrophil cytoplasmic antibody (ANCA). The increasing availability of the ANCA assay followed by characteristic biopsy has aided in the diagnosis of these rare conditions.

Untreated Wegener's granulomatosis/microscopic polyangiitis has a poor prognosis. For example, Wegener's granulomatosis has a mean survival of only five months with a one year survival of only $20 \% .^{1}$ In recent years, treatment with immunosuppression has radically altered prognosis. ${ }^{2}$

To highlight diagnosis and treatment in the elderly we report the successful treatment of three patients with ANCA-associated vasculitis aged 79,80 , and 80 years. We also review other published reports of treatment in the elderly and discuss complications of treatment with emphasis on elderly patients.

\section{Department of Medicine for the Elderly, Royal Bournemouth Hospital, Castle Lane East, Bournemouth BH7 7DW, UK S Sherwin V Fletcher P Medcalf}

Wessex Renal Unit, St Mary's Hospital, Portsmouth PO3 6AD, UK R Parry

Correspondence to Dr P Medcalf

Accepted 12 December 1995 polymyalgia rheumatica was made.

The patient showed an excellent sympto- matic, biochemical and immunological response to steroids. However, she developed persistent haematuria and proteinuria, with the presence of granular casts in her urine as her steroid dose was reduced. An intravenous urogram demonstrated no abnormality but a strongly positive cytoplasmic (c)ANCA level was detected in the serum. Renal biopsy performed after three months of steroid therapy showed glomerular changes with crescent formation and evidence of ischaemic collapse. A diagnosis of ANCA-associated vasculitis was made and prednisolone $40 \mathrm{mg}$ daily was restarted with the addition of cyclophosphamide at $100 \mathrm{mg} /$ day $(2 \mathrm{mg} / \mathrm{kg})$.

At six-months follow-up the patient remains symptomatically extremely well with normal renal function (creatinine of $79 \mu \mathrm{mol} / 1$ ), on prednisolone $10 \mathrm{mg}$ alternate days and cyclophosphamide $100 \mathrm{mg} /$ day.

\section{Case 2}

An 80-year-old woman with a long-standing history of joint pains, impaired hearing and a unilateral bloody nasal discharge presented with a three-week history of dyspnoea, haemoptysis and right-sided pleuritic chest pain.

Initial investigations revealed a mild microcytic anaemia with haemoglobin of $9.5 \mathrm{~g} / \mathrm{dl}$; mean corpuscular volume of $79.6 \mathrm{fl}$, and erythrocyte sedimentation rate of $118 \mathrm{~mm} / \mathrm{h}$. There was evidence of renal impairment with urea of $33.8 \mathrm{mmol} / \mathrm{l}$, creatinine $339 \mu \mathrm{mol} / \mathrm{l}$ and creatinine clearance $5.1 \mathrm{ml} / \mathrm{min}$. Urine examination revealed haematuria, proteinuria $(0.63 \mathrm{~g} / 24 \mathrm{~h})$ and the presence of granular casts. Small bilateral pleural effusions were present on the chest X-ray. Serum was strongly positive for cANCA. Renal histology showed a focal segmental necrotising glomerulonephritis with occasional crescents and a vasculitis consistent with Wegener's granulomatosis. A 1-cm diameter ulcerated area was seen on the right side of the nasal septum.

The patient's symptoms dramatically improved on prednisolone $40 \mathrm{mg} / \mathrm{day}$ and cyclophosphamide at $100 \mathrm{mg} /$ day.

At six months the patient remains well on cyclophosphamide $100 \mathrm{mg} /$ day and prednisolone $20 \mathrm{mg}$ alternate days, although a degree of renal failure persists (urea $14.1 \mathrm{mmol} / \mathrm{l}$, creatinine $225 \mu \mathrm{mol} / \mathrm{l}$ ).

\section{Case 3}

A 79-year-old man with a history of longstanding hypertension, joint pains, atypical chest pain and impaired hearing presented 
with a one-week history of severe polyarthralgia, haemoptysis, haematuria and a vasculitic rash.

Investigations demonstrated a haemoglobin of $12.5 \mathrm{~g} / \mathrm{dl}$, mean corpuscular volume of $91 \mathrm{fl}$, and an erythrocyte sedimentation rate of $59 \mathrm{~mm} / \mathrm{h}$. There was mild renal impairment with a urea of $8.1 \mathrm{mmol} / \mathrm{l}$, creatinine of $134 \mu \mathrm{mol} / 1$ and urinalysis showed blood and protein (less than $0.08 \mathrm{~g} / \mathrm{l}$ ). Linear subsegmental collapse at both bases was present on the chest X-ray.

An initial diagnosis of Wegener's granulomatosis/ANCA-positive vasculitis was made and prednisolone $40 \mathrm{mg} /$ day and cyclophosphamide $100 \mathrm{mg} /$ day were commenced. The circulating cANCA was strongly positive and renal biopsy was subsequently performed. This showed minor glomerulosclerosis only, however the biopsy was performed several days after starting steroid treatment, raising the possibility that histology was altered by therapy.

The patient showed a dramatic response with resolution of all his symptoms and an improvement both biochemically and immunologically.

At six months the patient remains well with minimal renal impairment (urea of $9.5 \mathrm{mmol} / \mathrm{l}$; creatinine of $127 \mu \mathrm{mol} / 1$ ) on prednisolone $10 \mathrm{mg}$ daily. Cyclophosphamide was stopped at two months post diagnosis because the patient felt it was making him unwell though there was no objective evidence of cyclophosphamide toxicity. He refused to take azathioprine.

\section{Discussion}

The indirect immunofluorescent ANCA assay gives two patterns; cANCA and pANCA (perinuclear staining). Wegener's granulomatosis is usually associated with cANCA, whilst microscopic polyangiitis is associated with pANCA. pANCA can also be seen in a wide variety of inflammatory disorders. ${ }^{3}$ In vasculitis, however, pANCA is usually associated with specificity for myeloperoxidase whereas this is not the case in inflammatory disorders and thus it is obligatory to perform an antimyeloperoxidase ELISA in association with ANCA immunofluorescence. Given its association with other disorders, the presence of ANCA is not enough to make the diagnosis of vasculitis. The diagnosis should be confirmed by biopsy. All our patients had cANCA on indirect immunofluorescence. In view of the absence of granulomatous change on biopsy, these patients have been given the diagnosis of 'ANCA associated vasculitis'. This is in accordance with recent consensus guidelines on vasculitis nomenclature. ${ }^{4}$

Published experience on vasculitis in the elderly is limited. The British Thoracic Society Research Committee performed a survey of Wegener's granulomatosis in England, Scotland and Wales for cases between 1975 and $1985 . .^{5}$ In this study it was shown that the mean age of presentation of the disease was 47.5 years (SD 13.3 years). These findings are similar to those of studies performed in the US. $^{2}$ In recent years reports of successful treatment in elderly patients have been published. Garrett et al reviewed 30 patients from the Wessex region with cANCA-associated renal disease. ${ }^{6}$ The mean age of the patients was 59 years but $30 \%$ were over 70 years at presentation. They found that age was not a factor in patient prognosis. Gaskin and Pusey

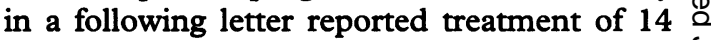
new patients over 70 years old. ${ }^{7}$ At one year $11 \stackrel{\hookrightarrow}{.}$ were still alive (79\%) compared to their overall $\overrightarrow{\vec{D}}$ one-year survival rate for 132 patients of $73 \%$. Briedigkeit $e t a l^{8}$ studied 64 patients from 든 1971-89 with an age range of 17-81 years. के They also found age was not an important $\stackrel{\square}{\square}$ factor in determining outcome. However, Jeffrey $e t a l^{9}$ in their five-year experience of 60 patients with a median age of 61 years, found a high early mortality in a subgroup of $\vec{\omega}$ patients who were all over 60 .

The paucity of data on vasculitis in the 8 elderly may reflect under-diagnosis and/or under-referral. Elderly patients present with $N$ multiple pathologies and atypical clinical symptoms and signs making the diagnosis of a rare systemic disorder more difficult. Renal involvement in vasculitis will give abnormal 을 findings on urinalysis (haematuria and proteinuria). Urinalysis is an important pointer to $\subseteq$ renal involvement and as such the importance of this simple test cannot be overemphasised. $\vec{\theta}$ The wide availability of the ANCA assay of should aid in diagonsis.

Treatment of Wegener's granulomatosis/ microscopic polyangiitis involves the use of immunosuppressive agents - usually cyclophosphamide in combination with corticoster- $\mathbb{D}$ oids. ${ }^{1,2,10}$ The largest reported experience is from the US National Institutes of Health. ${ }^{2}$ Their treatment protocol is shown in box 1 . In the UK the higher doses of cyclophosphamide are rarely used. For fulminant disease a course of plasma exchange or three doses of methyl prednisolone $(500 \mathrm{mg}$ or $1 \mathrm{~g}$ ) are given. The advantage of one over the other is not proven. ${ }^{11}$ Cyclophosphamide is usually substituted with azathioprine once in remission (usually three to six months). Long-term treatment is necessary

\section{NIH treatment protocol}

Induction

- cyclophosphamide $2 \mathrm{mg} / \mathrm{kg}$

- prednisolone $1 \mathrm{mg} / \mathrm{kg}$

Fulminant disease

- cyclophosphamide 3-5 mg/kg

- prednisolone $2-15 \mathrm{mg} / \mathrm{kg} 3$ days

\section{Maintenance}

- prednisolone for 4 weeks then changed to alternate day therapy over the next $1-3$ months. Then tapered to nil over the next 6-9 months

- cyclophosphamide for at least 1 year postremission then reduced by $25 \mathrm{mg}$ every $2-3$ months to zero 
in systemic vasculitis as the rate of relapse is as high as $33 \%^{12}$ or even $50 \% .^{2}$ Treatment is usually continued for at least one year post remission before tapering.

Drug toxicity is a major problem. Cyclophosphamide has well-documented side-effects, mainly involving the bone marrow, bladder and reproductive organs (box 2)., ${ }^{1,2,11}$ In the study of Hoffman et al, bladder cancer developed as early as seven months following treatment with cyclophosphamide while two patients developed lymphoma at 1.5 and 8.6 years and myelodysplasia occurred in three patients at 1.6, 9.6 and 13 years. Thus, the risk of malignancy is still a pertinent problem in the elderly.

Predisposition to infection is a significant problem. Hoffman et al found 73 patients (46\%) had episodes of serious infection over 1229 patient years. ${ }^{2}$ As well as bacterial infection, there was a 20 -fold increase in herpes zoster whilst on cyclophosphamide and prednisolone. Cyclophosphamide can predispose to infection by causing bone marrow suppression and neutropenia, and by interfering with lymphocyte function. Azathioprine predisposes less to malignancy and infection than cyclophosphamide. ${ }^{11,12}$ Prednisolone has been shown to be the most significant factor in cases of severe infection on immunosuppression. ${ }^{13}$ Fatal disease with cytomegalovirus (CMV) and Pneumocystis carinii has also been documented. ${ }^{13}$

Elderly patients have a reduced immune response, with both impairment of cellmediated immunity and a reduction in humoral immunity. ${ }^{14,15}$ Therefore elderly patients may be at greater risk from immunosuppression but may also need proportionally less in terms of type, dose, and duration.

The consequences of immunosuppression in the elderly have been looked at in the field of transplantation. ${ }^{15,16}$ Elderly patients have fewer rejection episodes with an increase in lifethreatening infection. Less immunosuppression is advocated, but no randomised controlled trials support this. Similarly there are no controlled trials comparing treatments of vasculitis in the elderly. In general terms, the use of the least toxic drugs for the shortest periods should be considered. This must be balanced with the high rate of relapse which would mean a repeat period of intense immunosuppression with associated risks. Therefore, switching from cyclophosphamide to azathioprine once in remission and early tapering of steroids should be considered. The associated use of antimicrobial prophylaxis (eg, cotrimoxazole and acyclovir or gancyclovir) should also be

1 Gaskin G, Pusey CD. In: Cameron S, Davison AM, Grünfeld JP, Kerr D, Ritz E, eds. Oxford textbook of clinical nephrology, 1st edn. Oxford: Oxford University Press, 1992; vol $1, \mathrm{pp} 612-36$.

2 Hoffman GS, Kerr GS, Leavitt RY, et al. Wegener Hoffman GS, Kerr GS, Leavitt RY, et al. Wegener
granulomatosis: an analysis of 158 patients. Ann Intern granulomatosis: an analysis

3 Kallenberg CG. Diversity of antigens recognized by ANCA-is this of clinical relevance? (editorial). Nephrol Dial Transplant 1994; 9: 228-9.

4 Jennette JC, Falk RJ, Andrassy K, et al. Nomenclature of systemic vasculitides. Proposal of an international consensus conference. Arthritis Rheum 1994; 37: 187-92.

\begin{tabular}{|l|}
\hline $\begin{array}{l}\text { Cyclophosphamide: side-effects } \\
\text { (from }\end{array}$ \\
\hline )
\end{tabular}

Box 2

\begin{tabular}{|l|}
\hline Learning points \\
\hline Diagnosis \\
- consider ANCA-associated vasculitis in the \\
elderly with multisystem disease \\
- do not forget simple urinalysis \\
- check serum ANCA \\
- biopsy of affected organ \\
Treatment \\
- feasible \\
- high incidence of side effects \\
- close monitoring essential \\
in remission \\
\end{tabular}

Box 3

considered, given the devastating effects of infection with CMV or $P$ carinii.

\section{Conclusion}

ANCA-associated vasculitis can be diagnosed and treated in the elderly. Diagnosis is aided by the widespread availability of the ANCA assay backed by biopsy, but the importance of bedside urinalysis should not be overlooked. Each of these patients has shown a good response to the combination of corticosteroid and cytotoxic therapy. Immunosuppression is feasible but carries a high risk of side-effects which may be higher in the elderly. More evidence is needed before specific recommendations can be made on doses, duration of treatment, and antimicrobial prophylaxis

We wish to thank Dr JC Mason and Dr JM Stevens for reading the manuscript and Miss $S$ Russell for her secretarial support.

5 Anderson G, Coles ET, Crane M, et al. Wegener's granuloma. A series of 265 British cases seen between 1975 and 1985 . A report by a sub-committee of the British Thoracic Society Research Committee. Of Med 1992; 83: Thoracic

6 Garrett PJ, Dewhurst AG, Morgan LS, Mason JC, Dathan

6 Garrett PJ, Dewhurst AG, Morgan LS, Mason JC, Dathan
JR. Renal disease associated with circulating antineutrophil cytoplasm activity. $Q \mathcal{F}$ Med 1992; 85: $731-49$.

7 Gaskin G, Pusey CD. ANCA-associated renal disease. (letter; comment). $Q \mathcal{F}$ Med 1993; 86: 138-9.

8 Briedigkeit L, Kettritz R, Gobel U, Natusch R. Prognostic factors in Wegener's granulomatosis. Postgrad Med F 1993, 69: $856-61$ 
9 Jeffrey RF, Gardiner DS, More IA, Junor BJ, Briggs JD. Crescentic glomerulonephritis: experience of a single

10 Falk RJ, Hogan S, Carey TS, Jennette JC. Clinical course of anti-neutrophil cytoplasmic autoantibody-associated glomerulonephritis and systemic vasculitis. The Glomerular Disease Collaborative Network. Ann Intern Med 1990; 113: 656-63.

11 Luqmani RA, Palmer RG, Bacon RA. Azathioprine, cyclophosphamide and chlorambucil. Baillieres Clin Rheucyclophosphamide and chlorambucil. Baillieres Clin Rheu-

12 Neild GH. Infectious complications in the management of systemic vasculitis and rapidly progressive glomerulonephritis. APMIS Suppl 1990; 19: 56-60.
13 Cohen J, Pinching AJ, Rees AJ, Peters DK. Infection and Immunosuppression. $Q \mathcal{F}$ Med 1982; 201: 1 - 15.

14 Fox RF. In: Evans JG, Williams TF, eds. Oxford textbook of geriatric medicine. Oxford: Oxford University Press, 1992; pp 51-6.

15 Ismail N, Hakim RM, Helderman JH. Renal replacement therapies in the elderly: Part II. Renal transplantation. $A m \mathcal{F}$ Kidney Dis 1994; 23: 1-15.

16 Morris GE, Jamieson NV, Small J, Evans DB, Calne R. Cadaveric renal transplantation in elderly recipients: is it Cadaveric renal transplantation in elderly recipients:
worthwhile? Nephrol Dial Transplant 1991; 6: 887-92. 